\title{
Factors triggering an additional resection and determining residual tumor volume on intraoperative MRI: analysis from a prospective single-center registry of supratentorial gliomas
}

\author{
Moritz Scherer, MD, ${ }^{1}$ Christine Jungk, MD, ${ }^{1}$ Alexander Younsi, MD, ${ }^{1}$ Philipp Kickingereder, MD, ${ }^{2}$ \\ Simon Müller, MSc, PhD, ${ }^{3}$ and Andreas Unterberg, MD, PhD' \\ ${ }^{1}$ Department of Neurosurgery and ${ }^{2}$ Division of Neuroradiology, Heidelberg University Hospital, Heidelberg; and ${ }^{3}$ Technology \\ Transfer Initiative, University of Stuttgart, Germany
}

\begin{abstract}
OBJECTIVE In this analysis, the authors sought to identify variables triggering an additional resection (AR) and determining residual intraoperative tumor volume in 1.5-T intraoperative MRI (iMRI)-guided glioma resections.

METHODS A consecutive case series of 224 supratentorial glioma resections (WHO Grades I-IV) from a prospective iMRI registry (inclusion dates January 2011-April 2013) was examined with univariate and multiple regression models including volumetric data, tumor-related, and surgeon-related factors. The surgeon's expectation of an AR, in response to a questionnaire completed prior to iMRI, was evaluated using contingency analysis. A machine-learning prediction model was applied to consider if anticipation of intraoperative findings permits preoperative identification of ideal iMRI cases.
\end{abstract}

RESULTS An AR was performed in $70 \%$ of cases after iMRI, but did not translate into an accumulated risk for neurological morbidity after surgery ( $p=0.77$ for deficits in cases with AR vs no AR). New severe persistent deficits occurred in $6.7 \%$ of patients. Initial tumor volume determined frequency of ARs and was independently correlated with larger tumor remnants delineated on iMRI $(p<0.0001)$. Larger iMRI volume was further associated with eloquent location $(p=0.010)$ and recurrent tumors $(p<0.0001)$, and with WHO grade $(p=0.0113)$. Greater surgical experience had no significant influence on the course of surgery. The surgeon's capability of ruling out an AR prior to iMRI turned out to incorporate guesswork (negative predictive value $43.6 \%$ ). In a prediction model, AR could only be anticipated with $65 \%$ accuracy after integration of confounding variables.

CONCLUSIONS Routine use of IMRI in glioma surgery is a safe and reliable method for resection guidance and is characterized by frequent ARs after scanning. Tumor-related factors were identified that influenced the course of surgery and intraoperative decision-making, and iMRI had a common value for surgeons of all experience levels. Commonly, the subjective intraoperative impression of the extent of resection had to be revised after iMRI review, which underscores the manifold potential of iMRI guidance. In combination with the failure to identify ideal iMRI cases preoperatively, this study supports a generous, tumor-oriented rather than surgeon-oriented indication for iMRI in glioma surgery.

http://thejns.org/doi/abs/10.3171/2015.11.FOCUS15542

KEY WORDS intraoperative magnetic resonance imaging; glioma; resection; additional resection; residual tumor

$\mathrm{S}$ INCE multiple studies have identified a maximized extent of resection (EOR) to be a predictor of prolonged patient survival in low-grade glioma (LGG) and high-grade glioma (HGG), the role of surgery has been accorded more value in contemporary glioma treatment regimens. ${ }^{6,9,15-18}$ Currently, a radical neurosurgical approach with gross-total resection (GTR) of the tumor is considered first-line therapy in resectable tumors. ${ }^{5}$ Without the aid of surgical guidance tools, however, the surgeon's perception of the EOR is often inaccurate, resulting in an undesired partial resection (PR) despite an intended GTR prior to surgery. ${ }^{12}$ In this regard, larger tumors, those

ABBREVIATIONS AIC = Akaike information criterion; $A R=$ additional resection; $A U C=$ area under the curve; EOR = extent of resection; $G T R=$ gross-total resection; HGG = high-grade glioma; iMRI = intraoperative MRI; LGG = low-grade glioma; NPV = negative predictive value; $P P V=$ positive predictive value; $P R=$ partial resection. ACCOMPANYING EDITORIAL DOI: 10.3171/2015.12.FOCUS15631.

SUBMITTED October 27, 2015. ACCEPTED November 30, 2015.

INCLUDE WHEN CITING DOI: 10.3171/2015.11.FOCUS15542. 
with proximity to eloquent areas, and deep-seated tumors have been shown to have a higher likelihood of being resected incompletely. ${ }^{1,12,19}$ Bringing together intraoperative MRI (iMRI) with multimodal neuronavigation forms a state-of-the-art concept for image-guided neurosurgery, facilitating the tightrope walk of achieving a radical but safe resection of gliomas. A proof of this concept has been delivered through a randomized controlled study in HGG by Senft et al. ${ }^{16}$ In LGG, a retrospective multicenter series of 288 cases indicated an independent association of high-field (1.5-T) iMRI guidance with the rate of GTR after surgery, without an accumulated risk of neurological deficits. ${ }^{3}$

Whereas many studies deal with preoperative planning, implementation of functional data into neuronavigation, and identification of risk factors for an unintended PR, few analyses have been dedicated to intraoperative decision making. ${ }^{413}$ Particularly in iMRI-guided surgery, the ability to delineate residual tumor and continue with the resection is a feature that might help to safely overcome limitations of conventional surgical techniques. The above-mentioned risk factors for unintended incomplete resections might not apply to iMRI cases and should be refined in this regard.

Moreover, the limited yet expanding availability of iMRI and the financial drawbacks of this method require careful and reasonable patient selection to tap the full potential of iMRI guidance in suitable patients. Allocation of cases to iMRI surgery is rarely based on scientific criteria, however. Identification of variables for residual tumor on iMRI and analysis of factors triggering an additional resection (AR) after intraoperative scanning can hypothetically allow preoperative identification of cases with high putative benefits of iMRI guidance. Furthermore, cases could be stratified in which iMRI would be considered optional (e.g., intended PR).

In this analysis, we sought to identify variables of an $\mathrm{AR}$ and residual tumor on high-field 1.5-T iMRI. A consecutive series of supratentorial glioma resections (WHO Grades I-IV) from a prospective iMRI registry was examined with univariate and multiple regression models for factors that influence surgical decision making during an iMRI-guided procedure. Moreover, a machine-learningbased prediction model with selected variables was applied to consider if anticipation of intraoperative findings permits preoperative identification of ideal iMRI cases.

\section{Methods}

\section{Prospective iMRI Registry}

High-field iMRI guidance is routinely applied in all patients with suspected glial tumors scheduled for tumor resection at the Neurosurgical Department at Heidelberg University Hospital. Since 2011, all iMRI procedures have been included in a prospective registry containing clinical and surgical as well as volumetric data. As a general principle, a maximal safe tumor resection with preservation of neurological and functional integrity is aspired to. A mainstay of the iMRI registry is the prospective definition of the goal of surgery, along with a volumetric definition of target resection volume prior to each procedure.

We identified 224 consecutive iMRI-guided glioma resections (WHO Grades I-IV) in 210 patients from our registry who were treated between January 2011 and April 2013, and we included those cases in this analysis of covariates of residual tumor on iMRI and AR. Written informed consent for iMRI-guided surgery was obtained preoperatively from all patients or an adequate representative, and the local ethics committee granted approval for retrospective analysis of prospective data.

\section{Standardized iMRI Procedure}

High-field 1.5-T iMRI is implemented into a 2-room, patient-to-the-magnet concept, initially described by Wirtz et al. at our department. ${ }^{20}$ Since 2009, a 1.5-T magnet has been used for intraoperative scans (Siemens Espree). The frequency of intraoperative scans is kept at a minimum (i.e., one per procedure) for reasons of time efficiency and accurate tissue contrast. Multimodal neuronavigation (Stryker Navigation System II iNtellect Cranial Navigation Software, Stryker) is implemented into every iMRI procedure. Initially, the surgeon attempts a maximized neuronavigated resection until the decision to perform an intraoperative scan is made. If residual tumor is delineated on iMRI, AR can be performed based on updated neuronavigation images.

\section{Volumetric Analysis and Prospective Definition of Surgical Goals}

The goal of surgery was defined by the surgeon as either GTR or PR. The feasibility of a safe GTR or maximized PR with preservation of neurological functionality was evaluated and judged individually by the surgeon prior to each procedure. Target resection volumes $\left(\mathrm{in}^{3}\right.$ ) based on preoperative imaging were manually segmented across all slices on axial planes by using the neuronavigation workstation. For high-grade tumors, contrast-enhanced T1-weighted sequences were used. In nonenhancing lowgrade tumors, T2-weighted FLAIR sequences were used. In intended GTR, all abnormal signal intensity on preoperative imaging was included for target resection volume. In intended PR, the definition of target resection volume was subject to the surgeon's individual evaluation of a maximized safe resection. If residual tumor was found on iMRI, manual segmentation was performed as described above to define target volumes for an AR. Postoperative imaging was volumetrically analyzed, with reviewers blinded to predefined resection targets.

\section{Additional Items in the iMRI Registry and Evaluation of ARs After iMRI}

Further elements in the iMRI registry included histology, tumor location and proximity to eloquent regions (defined as designated sensory-motor or speech areas), and involved deep white-matter tracts. Furthermore, postoperative neurological morbidity and functional outcome was evaluated for each patient. Postoperative deficits that had completely ceased at patient discharge were termed transient deficits. Minor persistent deficits were defined as residual symptoms present at 6-12 weeks after surgery that did not significantly affect a patient's independence in daily living. Severe persistent deficits were those that 
led to persistent impairment or dependency in daily living 6-12 weeks after surgery. The surgeon's experience was measured by the time that had passed since his or her board certification. Prior to iMRI, a questionnaire was completed to establish the surgeon's impression of EOR and the presumed odds for an AR on a 3-fold scale (1, exclusion of AR; 2, AR possible; or 3, planned AR). Expected rates of ARs were recorded and compared with observed rates after iMRI.

\section{Statistical Analysis}

All results are presented as the mean \pm SEM. Fisher's exact and chi-square tests were used for contingency analysis. One-way ANOVA was used for analysis of intergroup variance. Univariate screening was performed for variables of the outcome parameters AR after iMRI (binomial) and residual tumor volume on iMRI (continuous). For variable selection, logistic and linear regression models for each response variable were fit using a penalized maximum likelihood estimation. Guided by the Akaike information criterion (AIC), a fast backward variable selection procedure was applied to derive final models. We tested final models for each response variable on overfitting and bias by using internal validation through bootstrapping with 200 replications. The OR and regression coefficients are given with corresponding 95\% CI and 2-sided $\mathrm{p}$ value. For continuous variables (namely age, target volume, and residual tumor volume), the OR is reported for a change from 0.25 to 0.75 quantile. All continuous independent variables were tested on linearity. Finally, we used the selected variables in a random forest model followed by $10-$ fold cross-validation for prediction of the likelihood of an $\mathrm{AR}$ and residual tumor on postoperative MRI. Area under the curve (AUC) analysis was used to evaluate accuracy of predictions. Statistical analysis was performed with $\mathrm{R}$ (www.r-project.org, version 3.2.1), H20 (version 3.2.1), and PRISM (version 5.0c; GraphPad, Inc.) software. Values of $\mathrm{p}<0.05$ were regarded as statistically significant.

\section{Results}

\section{Patient Characteristics}

A total of 224 consecutive iMRI-guided glioma resections were retrieved from the registry. Patient characteristics are listed in Table 1. In 184 cases, the prospectively defined goal for surgery was GTR, and PR was aspired to in 40 cases. Tumor entities included 12 WHO Grade I (6 pilocytic astrocytomas, 4 gangliogliomas, 1 subependymoma, 1 neuroectodermal tumor); 31 WHO Grade II (16 astrocytomas, 8 oligodendrogliomas, 3 oligoastrocytomas, 4 others); 40 WHO Grade III (26 anaplastic astrocytomas, 13 anaplastic oligodendrogliomas, 1 anaplastic oligoastrocytoma); and 141 WHO Grade IV (138 glioblastomas, 2 gliosarcomas, 1 atypical teratoid/rhabdoid tumor) lesions. Of the 224 cases, $40.2 \%$ were recurrent tumors. Involvement of eloquent cortical areas or infiltration of deep white matter tracts was considered in 64/224 cases (28.6\%). The majority of intended PRs were performed in eloquent tumors (32/40 cases). Our series included 9 awake craniotomies.

Additional resections after iMRI were performed at
TABLE 1. Characteristics of 224 patients who underwent resections for supratentorial glioma

\begin{tabular}{|c|c|c|}
\hline Characteristic & Total, $n=224$ & $\%$ \\
\hline Age in yrs; mean \pm SEM & $50.9 \pm 17.1$ & \\
\hline Sex (no. M/F) & $151 / 73$ & \\
\hline \multicolumn{3}{|l|}{ Tumor entity } \\
\hline \multicolumn{3}{|l|}{ WHO Grade } \\
\hline 1 & 12 & 5.4 \\
\hline$\|$ & 31 & 13.8 \\
\hline III & 40 & 17.9 \\
\hline IV & 141 & 62.9 \\
\hline Recurrent tumors & 90 & 40.2 \\
\hline Tumor laterality (no. It/rt) & $116 / 108$ & \\
\hline \multicolumn{3}{|l|}{ Tumor location } \\
\hline Frontal & 84 & 37.5 \\
\hline Temporal & 69 & 30.8 \\
\hline Parietal & 43 & 19.2 \\
\hline Occipital & 23 & 10.3 \\
\hline Intraventricular & 5 & 2.2 \\
\hline Eloquent location & 64 & 28.6 \\
\hline \multicolumn{3}{|l|}{ Surgery } \\
\hline Intended GTR & 184 & 82.1 \\
\hline Intended PR & 40 & 17.9 \\
\hline AR after iMRI & 156 & 69.6 \\
\hline Residual tumor present after surgery & 73 & 32.6 \\
\hline \multicolumn{3}{|l|}{ Neurological deficits } \\
\hline No new deficits & 157 & 70.1 \\
\hline Transient deficits & 30 & 13.4 \\
\hline Mild persistent deficits & 22 & 9.8 \\
\hline Severe persistent deficits & 15 & 6.7 \\
\hline
\end{tabular}

an equal rate of $70 \%$ in both subgroups of intended GTR and intended PR $(p=1.0)$. Volumetric analysis for final resection results was performed in intended GTR cases. The mean residual tumor volume after surgery was 0.13 $\pm 0.04 \mathrm{~cm}^{3}$ in cases in which GTR was intended, which correlates to a final EOR of $99.6 \% \pm 0.1 \%$. A GTR was achieved in 151/184 cases in which it was planned (82.1\%). See Table 2 for detailed volumetric analysis stratified for WHO grades.

Surgical procedures were performed by 9 board-certified neurosurgeons of various experience levels, which was graded according to time since their board examinations. Senior surgeons performed 58.9\% (132/224) of surgeries and had a mean experience of $145.3 \pm 1.9$ months when performing surgery in this series $(>10$ years of experience). Attending surgeons with an intermediate experience level treated $24.1 \%$ (54/224) of cases, with a mean experience of $44.5 \pm 1.5$ months ( $2-5$ years). Junior staff with a mean experience of $14.3 \pm 1.2$ months $(<2$ years) were responsible in $17.0 \%(38 / 224)$ of cases.

\section{Clinical Outcome After iMRI-Guided Surgery}

There was no technology-related morbidity or surgeryrelated mortality observed in this cohort. At discharge, 
TABLE 2. Volumetric analysis in patients with supratentorial glioma

\begin{tabular}{|c|c|c|c|c|c|c|c|c|}
\hline \multirow{2}{*}{$\begin{array}{l}\text { WHO } \\
\text { Grade }\end{array}$} & \multirow[b]{2}{*}{ No. } & \multirow{2}{*}{$\begin{array}{l}\text { Intended } \\
\text { GTR/PR }\end{array}$} & \multirow{2}{*}{$\begin{array}{l}\text { Target Resection Vol; } \\
\text { Mean } \pm \text { SEM }\left(\mathrm{cm}^{3}\right)\end{array}$} & \multicolumn{2}{|c|}{$A R$} & \multicolumn{2}{|c|}{ Residual Tumor Vol on iMRI } & \multirow{2}{*}{$\begin{array}{l}\text { Residual Tumor Postop; No. } \\
\text { in Intended GTR/PR Group* }\end{array}$} \\
\hline & & & & No. & $\%$ & Mean \pm SEM $\left(\mathrm{cm}^{3}\right)$ & $\%$ of Initial Vol \pm SEM & \\
\hline I & 12 & $011 / 1$ & $10.94 \pm 2.50$ & 4 & 33.3 & $0.30 \pm 0.17$ & $06.6 \pm 4.0$ & $00 / 1$ \\
\hline II & 31 & $023 / 8$ & $29.47 \pm 5.77$ & 25 & 80.7 & $3.86 \pm 0.75$ & $16.7 \pm 3.3$ & $03 / 8$ \\
\hline III & 40 & 030/10 & $31.24 \pm 5.51$ & 29 & 72.5 & $4.54 \pm 1.18$ & $19.2 \pm 4.6$ & $03 / 10$ \\
\hline IV & 141 & $120 / 21$ & $33.32 \pm 2.38$ & 98 & 69.5 & $2.78 \pm 0.47$ & $08.5 \pm 1.2$ & $27 / 21$ \\
\hline Total & 224 & $184 / 40$ & $31.27 \pm 1.98$ & 156 & 69.6 & $3.16 \pm 0.38$ & $11.5 \pm 1.2$ & $33 / 40$ \\
\hline
\end{tabular}

* In contrast-enhancing tumors, GTR is defined as removal of all tumor with contrast enhancement. In nonenhancing lesions, GTR is defined as removal of all areas of FLAIR hyperintensity.

$83.5 \%$ of patients were in unchanged neurological condition and without any new deficits after surgery. Transient neurological deficits were observed in $13.4 \%$ of cases immediately after surgery, which had completely ceased at discharge from hospital. New severe persistent neurological deficits occurred in $6.7 \%$ of patients; these were defined as deficits with impairment in activities of daily living > 6-12 weeks after surgery (see Table 1). Table 3 provides a detailed analysis of postoperative morbidity, with special focus on motor and language function in relation to an AR after iMRI. An AR did not lead to an accumulation of either transient, minor, or severe persistent neurological deficits postoperatively, compared with cases in which no AR was performed $(\mathrm{p}=0.77)$. Furthermore, the mean amount of residual tumor volume on iMRI was not a significant determinant of either transient, minor, or severe persistent neurological deficits after surgery $(\mathrm{p}=$ 0.74 ). Surgical experience had no significant effect on neurological morbidity; the occurrence of postoperative deficits was comparable for junior, intermediate, and senior surgeons $(\mathrm{p}=0.71)$.

No difference in rate of postoperative neurological deficits was observed between intended GTR and intended PR subgroups $(p=0.94)$. Neither WHO grade $(p=0.43)$ nor initial tumor size $(\mathrm{p}=0.35)$ predicted new neurological morbidity. However, a strong trend was observed for accumulation of new postoperative deficits in eloquently located tumors $(\mathrm{p}=0.0575)$.

\section{Univariate Screening for Variables}

Results from univariate screening for variables of an $\mathrm{AR}$ and residual tumor volume on iMRI are listed in Table 4. Sex and WHO grade had an association with ARs, whereas neither tumor laterality nor the primary tumor location were significant predictors for an AR. Eloquent tumors had AR rates comparable to noneloquent tumors, but residual volume on iMRI was significantly larger in the former. Similarly, the preoperative goal of surgery did not significantly influence rates of ARs, but residual tumor volume was larger in intended PR. Initial tumor volume was positively associated with both ARs and residual tumor on iMRI. Remarkably, surgical experience had an influence on neither the rate of AR nor on the amount of residual tumor to be removed after iMRI.

\section{Multiple Regression Model for Identification of Variables}

All variables from univariate screening in Table 4 were transferred into a multiple regression model, where we applied an AIC-guided fast backward selection procedure. The regression models were validated by internal bootstrapping with 200 replications. Internal bootstrap validation showed that our models neither had biased coefficients, nor were they overfitted. Results from multiple variable selection are given for ARs and for residual tumor volume on iMRI (Table 5).

\section{Influence of Initial Tumor Size}

The initial size of the tumor had an impact on the frequency of AR after iMRI and also in multiple regression analysis. Moreover, we found an independent positive correlation of initial tumor volume with residual tumor volume on iMRI (OR 2.61, 95\% CI 1.88-3.33, p < 0.0001) (Table 5). After AR during surgery, however, initial tumor volume was not associated with postoperative residual tumor in multiple regression analysis (OR 1.36, 95\% CI $0.87-2.13, \mathrm{p}=0.17$ ).

\section{Influence of Tumor Location}

Tumor location according to its hemisphere or lobe, or in combination, had no significant impact on iMRI findings (Table 4). When a tumor was considered eloquent, this had an independent effect on resection strategy, with a significantly larger residual tumor delineated on iMRI $(\mathrm{OR} 1.70,95 \%$ CI $0.41-2.99, \mathrm{p}=0.010)$ (Table 5).

\section{Influence of Tumor Entity}

The WHO grade had no independent association with ARs in our cohort. Compared with WHO Grade IV tumors, however, the amount of residual tumor on iMRI was significantly larger in WHO Grade II gliomas (OR 2.52, $95 \%$ CI 0.98-4.06, p = 0.011) (Tables 2 and 5). Resection of recurrent lesions did not lead to an accumulated risk of ARs, but intraoperative residual tumor volume was also significantly larger in these cases (OR 2.67, 95\% CI 1.44$3.90, \mathrm{p}<0.0001)$ (Table 5).

\section{Influence of Surgical Experience and Perception of EOR}

In our multiple regression model, the experience of the surgeon had no significant effect on rates of ARs and residual volume delineated on iMRI. The surgeon's presumed odds for an AR were queried based on an increasing scale of 1 (exclusion of AR) to 3 (planned AR), and showed a strong and independent correlation with observed rates of $\mathrm{AR}$ and also residual tumor volume on iMRI in univariate and multiple regression analysis. Moreover, increasing 
TABLE 3. Postoperative morbidity in patients with supratentorial glioma*

\begin{tabular}{|c|c|c|c|c|c|c|}
\hline \multirow[b]{2}{*}{ Type of Deficit } & \multicolumn{2}{|c|}{ Transient Deficits } & \multicolumn{2}{|c|}{ Minor Persistent Deficits } & \multicolumn{2}{|c|}{ Severe Persistent Deficits } \\
\hline & AR & No AR & AR & No AR & AR & No AR \\
\hline Motor & 7 & 2 & 7 & 3 & 10 & 3 \\
\hline Language & 11 & 4 & 4 & 4 & 2 & 0 \\
\hline Other & 3 & 3 & 3 & 0 & 0 & 0 \\
\hline Subtotal & $21(13.5 \%)$ & $9(13.2 \%)$ & $14(9.0 \%)$ & $7(10.3 \%)$ & $12(7.7 \%)$ & $3(4.4 \%)$ \\
\hline Total & \multicolumn{2}{|c|}{$30(13.4 \%)$} & \multicolumn{2}{|c|}{$21(9.4 \%)$} & \multicolumn{2}{|c|}{$15(6.7 \%)$} \\
\hline
\end{tabular}

* Postoperative morbidity after iMRI-guided surgery; the heading "No AR" denotes no additional resection after iMRI. Transient deficits had completely ceased at patient discharge. Minor persistent deficits remained after $>3$ months and did not affect daily living. Severe deficits led to persistent impairment after surgery. Other deficits included pure sensory or visual impairment.

ORs indicate the surgeon's ability to correctly estimate the greater likelihood for an AR and to anticipate greater residual tumor on iMRI (Tables 4 and 5). In cross-table calculations, we further compared expected rates of ARs with observed rates of ARs after iMRI. The positive predictive value (PPV) of the surgeon's expectation, or the proportion of cases in which ARs were performed when they were also expected, was high (PPV 93.1\%, 95\% CI $77.2 \%-99.15 \%$ ). In turn, the ability to sustainably exclude ARs from the intraoperative impression was very low. In other words, low negative predictive value (NPV) of the surgeon's perception (NPV 43.6\%, 95\% CI 34.2\%-53.4\%)

TABLE 4. Univariate screening for variables in patients with supratentorial glioma*

\begin{tabular}{|c|c|c|c|c|c|c|c|c|}
\hline \multirow[b]{2}{*}{ Variable } & \multicolumn{4}{|c|}{ AR } & \multicolumn{4}{|c|}{ Residual Tumor Vol on iMRI } \\
\hline & OR & $\begin{array}{c}\text { Lower } \\
95 \%\end{array}$ & $\begin{array}{c}\text { Upper } \\
95 \%\end{array}$ & $p$ Value & Effect & Lower 95\% & Upper 95\% & $\mathrm{p}$ Value \\
\hline Sex (F vs M) & 2.81 & 1.39 & 5.69 & 0.0040 & 1.00 & -0.59 & 2.60 & 0.2163 \\
\hline Age & 1.11 & 0.74 & 1.67 & 0.6207 & -0.63 & -1.68 & 0.42 & 0.2404 \\
\hline \multicolumn{9}{|l|}{ Tumor location } \\
\hline Frontal & Reference & & & 0.1440 & Reference & & & \\
\hline Temporal & 0.68 & 0.33 & 1.38 & & -1.32 & -3.14 & 0.49 & 0.2978 \\
\hline Parietal & 0.57 & 0.25 & 1.28 & & -1.00 & -3.09 & 1.09 & \\
\hline Occipital & 1.14 & 0.38 & 3.47 & & -2.00 & -4.58 & 0.57 & \\
\hline Intraventricular & 0.08 & 0.01 & 0.75 & & -3.90 & -8.93 & 1.13 & \\
\hline Laterality (It vs rt) & 1.52 & 0.85 & 2.72 & 0.1586 & 0.44 & -1.05 & 1.93 & 0.5599 \\
\hline Eloquent location (yes vs no) & 0.82 & 0.44 & 1.54 & 0.5412 & 2.97 & 1.36 & 4.57 & 0.0003 \\
\hline \multicolumn{9}{|l|}{ WHO grade } \\
\hline 1 & 0.22 & 0.06 & 0.78 & 0.0261 & -2.49 & -5.89 & 0.91 & 0.0846 \\
\hline$\|$ & 2.22 & 0.80 & 6.18 & & 1.13 & -1.06 & 3.32 & \\
\hline III & 1.33 & 0.60 & 2.96 & & 1.80 & -0.19 & 3.79 & \\
\hline IV & Reference & & & & Reference & & & \\
\hline Recurrent tumor (yes vs no) & 0.73 & 0.41 & 1.31 & 0.2895 & 1.37 & -0.14 & 2.89 & 0.0756 \\
\hline Goal of surgery (PR vs GTR) & 0.98 & 0.47 & 2.08 & 0.9671 & 4.97 & 3.13 & 6.82 & $<0.0001$ \\
\hline Initial tumor vol & 1.84 & 1.18 & 2.87 & 0.0073 & 3.15 & 2.38 & 3.92 & $<0.0001$ \\
\hline \multicolumn{9}{|l|}{ Surgeon's experience† } \\
\hline 1 & 1.23 & 0.53 & 2.88 & 0.8495 & -0.82 & -2.98 & 1.34 & 0.7560 \\
\hline 2 & 1.16 & 0.57 & 2.33 & & -0.21 & -2.02 & 1.60 & \\
\hline 3 & Reference & & & & Reference & & & \\
\hline \multicolumn{9}{|l|}{ Surgeon's odds for AR $\ddagger$} \\
\hline 1 & Reference & & & 0.0001 & Reference & & & $<0.0001$ \\
\hline 2 & 3.50 & 1.72 & 7.11 & & 1.87 & 0.37 & 3.37 & \\
\hline 3 & 10.23 & 2.32 & 45.20 & & 7.71 & 5.60 & 9.82 & \\
\hline
\end{tabular}

* Boldface type indicates statistical significance.

† Surgeon's experience: 1 , junior staff; 2 , intermediate experience; 3 , senior staff.

$\ddagger$ Surgeon's odds for an AR were categorized according to a 3-fold scale of the surgeon's estimated likelihood of AR queried prior to iMRI: 1, exclusion of AR; 2, AR possible; 3, planned AR. 
TABLE 5. Multiple regression model for identification of variables for AR and residual tumor volume on iMRI obtained in patients with supratentorial glioma *

\begin{tabular}{|c|c|c|c|c|c|c|c|c|}
\hline \multirow[b]{2}{*}{ Variable } & \multicolumn{4}{|c|}{$\mathrm{AR}$} & \multicolumn{4}{|c|}{ Residual Tumor Volume on iMRI } \\
\hline & OR & Lower 95\% & Upper 95\% & p Value & Effect & Lower $95 \%$ & Upper 95\% & $p$ Value \\
\hline Initial tumor vol & 1.36 & 0.87 & 2.13 & 0.1731 & 2.61 & 1.88 & 3.33 & $<0.0001$ \\
\hline Sex (F vs M) & 2.49 & 1.22 & 5.08 & 0.0124 & 1.29 & 0.01 & 2.58 & 0.0487 \\
\hline \multicolumn{9}{|l|}{ Surgeon's odds for AR† } \\
\hline 1 & Reference & & & 0.0009 & Reference & & & 0.0001 \\
\hline 2 & 2.99 & 1.49 & 5.99 & & 0.85 & -0.42 & 2.12 & \\
\hline 3 & 4.86 & 1.61 & 14.64 & & 4.17 & 2.35 & 5.99 & \\
\hline Eloquent location (yes vs no) & & & & & 1.70 & 0.41 & 2.99 & 0.0100 \\
\hline Recurrent tumor (yes vs no) & & & & & 2.67 & 1.44 & 3.90 & $<0.0001$ \\
\hline \multicolumn{9}{|l|}{ WHO grade } \\
\hline 1 & & & & & -0.97 & -3.55 & 1.61 & 0.0113 \\
\hline II & & & & & 2.52 & 0.98 & 4.06 & \\
\hline III & & & & & -0.45 & -2.22 & 1.31 & \\
\hline IV & & & & & Reference & & & \\
\hline
\end{tabular}

* Boldface type indicates statistical significance. Note: to achieve the best possible regression fit, multiple regression selection also includes nonsignificant variables under the condition that they improve the fit of the model. All variables stated have relevant impact for outcome measures. Models were validated by 200 -replicate bootstrapping.

† Based on a 3-fold scale of the surgeon's estimated likelihood of AR queried prior to iMRI: 1, exclusion of AR; 2, AR possible; 3, planned AR.

indicates that ARs were performed contrary to the surgeon's expectation in $56 \%$ of cases. However, NPV increased with surgical experience. Whereas the NPV of intraoperative assessment for an AR was 26.7\% (95\% CI $7.8 \%-55.1 \%$ ) in junior surgeons, it increased to $38.2 \%$ (95\% CI 22.2\%-56.4\%) in surgeons of intermediate experience, and reached $50.0 \%$ (95\% CI $36.6 \%-63.4 \%$ ) in senior staff.

\section{Model-Based Prediction of an AR}

To test whether AR could reliably be predicted according to the variables identified through multiple regression analysis (Table 5), a random forest learning algorithm was trained and tested in a 10-fold cross-validation. In AUC analysis for prediction accuracy, the random forest algorithm yielded an AUC value of $65.0 \%$ (95\% CI $49.7 \%$ $80.2 \%$ ), suggesting that predictions were correct in only two-thirds of cases.

\section{Discussion}

In this study we analyzed a consecutive series of highfield 1.5-T iMRI-guided glioma resections from a prospective registry. We sought to identify variables associated with additional resections and residual tumor volume on iMRI, and evaluated whether these factors are suitable to identify ideal iMRI cases preoperatively. We found that ARs were frequently performed after iMRI in $70 \%$ of cases in our series, regardless of whether GTR or PR was intended. Additional resections and extension of surgery performed after iMRI did not lead to new postoperative neurological morbidity. Initial tumor size determined frequency of ARs and was independently correlated with larger tumor remnants delineated on iMRI. Greater surgical experience was not associated with reduced rates of
ARs or smaller tumor volume on iMRI. Regarding preoperative identification of cases that would need iMRI, AR could only be predicted with $65 \%$ accuracy by an algorithm after integration of identified variables of AR.

\section{High Rates of ARs and Impact on Clinical Outcome}

In our analysis of 224 consecutive high-field iMRI cases, we observed a $70 \%$ rate of AR after iMRI. This is in contrast to rates of $25 \%-52 \%$ reported in other iMRI series of mixed glioma entities. ${ }^{2,47,10,16,21}$ Inevitably, the fact that all resections in our study were scheduled with iMRI imposed a treatment bias and influenced the surgeon in his or her decision when to scan during surgery. We did not observe signs of premature scanning in our data, however. First, the mean AR volume was very small $(3.16 \pm$ $0.38 \mathrm{~cm}^{3}$ ) (Table 2), suggesting that in general, only small amounts of residual tumor were removed after iMRI. In our study, we regard ARs as a way of optimizing the completeness of the resection, which has been shown to have an impact on survival. ${ }^{11,15,17}$ Second, we specifically designed our surgeon questionnaire to evaluate the impact of treatment bias. Incorporating this information, premature scans were limited to only 29/224 cases (13\%) where AR was planned before obtaining iMRI. This leaves a big share of cases in which the surgeon was unsure or even planned to terminate surgery before iMRI was acquired. The potential benefit of iMRI guidance for optimizing EOR is especially present in these cases.

Although the value of iMRI in intended PRs is controversial, rates of AR were equally high in intended GTR and PR in our series, suggesting a mutual benefit of iMRI. ${ }^{2,8}$ However, there is an obvious selection bias to this finding: of 40 cases of intended PR, 32 tumors were located in proximity to eloquent areas or infiltrated central brain structures. In these cases, the goal of surgery was to 
achieve a radical resection with deliberate sparing of distinct tumor areas, instead of mere tumor debulking. In this constellation of oncological goals and anatomical boundaries, iMRI guidance was equally helpful in intended PR. In our descriptive data, the value of iMRI guidance arises mainly from the augmented precision of neuronavigation during surgery.

\section{Postoperative Morbidity}

Overall rates of new persistent deficits (16.5\%) were elevated when compared with other iMRI series in the field, which report a 5\%-13\% incidence. Partially, this is explained by also including $9.8 \%$ of minor persistent deficits in the evaluation, in addition to any significant impairment reported by the patients at follow-up (Table 3). Severe persistent and impairing deficits were observed in $6.7 \%$, which is in line with published morbidity rates after surgery. ${ }^{3,4,7,16,21}$ However, direct comparability to other published series is limited due to our inhomogeneous cohort of mixed tumor entities, locations, and sizes. A trend toward a higher rate of deficits in eloquent tumors also suggests selection bias that will have to be addressed in a separate analysis of neurological outcome after iMRI surgery. Above all, our analysis did not reveal any statistically significant correlation between ARs or the amount of residual intraoperative tumor and the appearance of new postoperative neurological deficits. Moreover, surgical experience did not affect postoperative morbidity, which recalls the safety aspect of iMRI guidance previously acknowledged in the literature, and illustrates that extension of surgery after iMRI was not performed at the cost of functionality in this series. . $^{3,4,7,10,16}$

\section{Influence of Initial Tumor Volume on AR and Residual Volume}

In univariate and multiple regression analysis, initial tumor volume was the only variable that concordantly affected rate of ARs and amount of residual tumor on iMRI (Tables 4 and 5). This finding reflects the everyday perception of neurosurgeons that larger tumors are more difficult to resect due to limited exposure and visual control. ${ }^{12,19}$ An association of initial volume with AR has also been shown in a previous iMRI study, suggesting a cutoff volume of $20 \mathrm{~cm}^{3}$ for significantly higher rates. ${ }^{2}$ In our series we observed AR in $>50 \%$ of cases, even in smaller tumors $\left(\leq 10 \mathrm{~cm}^{3}\right.$ ), reflecting the potential for improving resection results irrespective of size cutoffs. Most importantly, initial volume was not a significant variable for residual tumor present on postoperative imaging. This finding illustrates how certain limiting factors for radical surgery, like initial tumor size, can indeed be overcome with the technical assistance of iMRI and reflects a key advantage of this method. ${ }^{12,19}$

\section{Influence of Tumor Location, Eloquence, and WHO Grade}

A limiting factor for EOR that cannot be approached with iMRI guidance alone is tumor eloquence. Awake craniotomy and/or electrophysiology assessments with stimulation and mapping can be applied in combination to iMRI to safely delineate nonfunctional areas for resection in eloquent tumors. ${ }^{13,14}$ We observed an independent association with higher residual tumor volume on iMRI in eloquent tumors (OR 1.7, p =0.010) (Table 5). This probably reflects a more defensive resection when approaching eloquent areas, until iMRI provides reassurance of tumor boundaries. Another finding that reflects the everyday struggle with visual tumor delineation is the independent association of recurrent tumor (OR 2.67, p < 0.0001) and WHO Grade II tumors (OR 2.52, $\mathrm{p}=0.01$ for WHO Grade II vs IV) with greater amounts of residual tumor on iMRI. Especially in LGGs (WHO Grade II), where infiltrative growth patterns render visual and haptic delineation exceedingly difficult, iMRI can provide an unparalleled capability in precisely depicting residual tumor. This demand for additional feedback in WHO Grade II tumors is clearly reflected in our findings.

Although an effect of the above-mentioned variables on residual tumor volume could be identified, this did not apply to the rate of ARs accordingly. Mainly, high rates of ARs throughout all tumors in this study explain why the impact of eloquent location, recurrent tumor, and WHO grade on frequency of ARs failed to reach statistical significance. From another point of view, this could reflect a strong subjective component influencing decision making during surgery, even with adjunct iMRI guidance. Nevertheless, we observed the highest rates of ARs (exceeding $80 \%$ ) in WHO Grade II tumors in this series (Table 2).

\section{Influence of Surgeon and Perception of EOR}

Surgical experience affected neither the rate of AR nor the amount of residual tumor on iMRI in univariate and multiple regression analysis (Table 4). This has previously been proposed by other series and supports the value of iMRI for junior as well as senior neurosurgeons. ${ }^{2,16}$ To corroborate the value of iMRI for intraoperative decision making, we questioned the surgeon about the anticipated further resection process before he or she looked at the iMRI. The visual intraoperative impression of EOR is known to be commonly overestimated, which was clearly confirmed by our data.,12 Assumptions about the EOR prior to iMRI had to be revised in the majority of cases after intraoperative scanning. On the one hand, surgeons were indeed able to gradually anticipate residual tumor on $\mathrm{iMRI}$ and ARs from their intraoperative impression, meaning that their perception had a high PPV (93.1\%). On the other hand, ARs were performed contrary to the surgeon's expectation in $56.4 \%$ of cases. The poor ability to sustainably exclude an AR from the intraoperative impression is reflected by low NPVs $(43.6 \%)$ that diminish the surgeon's perception to mere guesswork. We saw a gradual increase in the ability to correctly evaluate the progress of the resection and to exclude ARs with greater accuracy along with increasing surgical experience. Nevertheless, accuracy remained poor, with NPVs never exceeding $50 \%$, not even in senior surgeons with experience of more than 10 years in glioma surgery.

Our results indicate a big discrepancy between the surgeon's perception as opposed to the true EOR visualized by iMRI. This clearly justifies the implementation of intraoperative imaging in general and iMRI in particular to increase consistency in glioma EOR in the light of survival benefits conveyed by this approach. Furthermore, omission of iMRI guidance for experienced surgeons or presumed 
straightforward tumor resections cannot be supported by our data. Rather, the uniform value of iMRI guidance for surgeons of all experience levels advocates the use of iMRI as a learning tool and as a measure of quality control. Through its immediate feedback about intraoperative EOR, iMRI can provide an optimized learning curve in junior surgeons while assuring uncompromised resection results comparable to senior colleagues. Moreover, evaluation of the precise location of intraoperative tumor remnants within the resection cavity is an interesting aspect for future studies that could transfer unique knowledge from iMRI-guided surgery to non-iMRI-guided surgery. Identification of hot spots for tumor remnants will be of great interest for all glioma surgeons, regardless of the guidance technique used.

\section{Predicting ARs}

Finally, we sought to test whether ARs after iMRI can be predicted, which would allow for a preoperative identification of ideal cases in which there is greater potential benefit from iMRI surgery. With variables identified in our AIC-guided multiple regression model (Table 5), we trained a random forest learning algorithm for prediction of AR. The AUC values of $65 \%$ (95\% CI 49.7\%-80.2\%) derived from cross-validation were inconclusive, however, suggesting correct predictions in only two-thirds of cases. Moreover, the $95 \%$ CI also incorporated the 50\% accuracy level, which implies that our predictions are comparable to a coin flip. Consequently, accuracy of predictions did not permit reliable preoperative identification of ideal iMRI cases, which supports our current approach to generously use iMRI guidance in all glioma resections. We again see a strong subjective component that influences decision making for AR and prevents the algorithm from generating robust predictions.

\section{Conclusions}

Routine use of high-field iMRI in glioma surgery is a safe and reliable method for resection guidance, and is characterized by frequent ARs after scanning. Initial tumor volume is associated with the frequency of ARs and is an independent variable of the amount of residual tumor delineated on iMRI, along with eloquent location and WHO grade. Information derived from iMRI is shown to be useful for surgeons of all experience levels, with frequent revision of their intraoperative perception of EOR after iMRI results. The factors influencing the intraoperative decision to continue resection are manifold and cannot be anticipated from preoperative data with sufficient accuracy to allow preoperative identification of ideal iMRI cases. The combination of numerous unexpected iMRI findings and surgeon-independent frequency of iMRI-influenced decisions depicts the broad potential of iMRI and supports a generous tumor-oriented rather than surgeon-oriented indication in glioma surgery.

\section{Acknowledgments}

We thank MRI technicians Bernhard Beigel and Vitali Abb for assistance in MRI procedures, data collection, and registry maintenance.

\section{References}

1. Albert FK, Forsting M, Sartor K, Adams HP, Kunze S: Early postoperative magnetic resonance imaging after resection of malignant glioma: objective evaluation of residual tumor and its influence on regrowth and prognosis. Neurosurgery 34:45-61, 1994

2. Bohinski RJ, Kokkino AK, Warnick RE, Gaskill-Shipley MF, Kormos DW, Lukin RR, et al: Glioma resection in a shared-resource magnetic resonance operating room after optimal image-guided frameless stereotactic resection. Neurosurgery 48:731-744, 2001

3. Coburger J, Merkel A, Scherer M, Schwartz F, Gessler F, Roder C, et al: Low-grade glioma surgery in intraoperative MRI: Results of a multi-center retrospective assessment. Neurosurgery [epub ahead of print], 2015

4. Hatiboglu MA, Weinberg JS, Suki D, Rao G, Prabhu SS, Shah K, et al: Impact of intraoperative high-field magnetic resonance imaging guidance on glioma surgery: a prospective volumetric analysis. Neurosurgery 64:1073-1081, 2009

5. Hervey-Jumper SL, Berger MS: Role of surgical resection in low- and high-grade gliomas. Curr Treat Options Neurol 16:284, 2014

6. Kreth FW, Thon N, Simon M, Westphal M, Schackert G, Nikkhah G, et al: Gross total but not incomplete resection of glioblastoma prolongs survival in the era of radiochemotherapy. Ann Oncol 24:3117-3123, 2013

7. Kuhnt D, Ganslandt O, Schlaffer SM, Buchfelder M, Nimsky C: Quantification of glioma removal by intraoperative highfield magnetic resonance imaging: an update. Neurosurgery 69:852-863, 2011

8. Livne O, Harhel R, Hadani M, Spiegelmann R, Feldman Z, Cohen ZR: Intraoperative magnetic resonance imaging for resection of intra-axial brain lesions. A decade of experience using low-field magnetic resonance imaging, Polestar N-10, 20,30 systems. World Neurosurg 82:770-776, 2014

9. McGirt MJ, Chaichana KL, Gathinji M, Attenello FJ, Than $\mathrm{K}$, Olivi A, et al: Independent association of extent of resection with survival in patients with malignant brain astrocytoma. J Neurosurg 110:156-162, 2009

10. Nimsky C, Fujita A, Ganslandt O, Von Keller B, Fahlbusch $\mathrm{R}$ : Volumetric assessment of glioma removal by intraoperative high-field magnetic resonance imaging. Neurosurgery 55:358-371, 2004

11. Oppenlander ME, Wolf AB, Snyder LA, Bina R, Wilson JR, Coons SW, et al: An extent of resection threshold for recurrent glioblastoma and its risk for neurological morbidity. J Neurosurg 120:846-853, 2014

12. Orringer D, Lau D, Khatri S, Zamora-Berridi GJ, Zhang K, Wu C, et al: Extent of resection in patients with glioblastoma: limiting factors, perception of resectability, and effect on survival. J Neurosurg 117:851-859, 2012

13. Ottenhausen M, Krieg SM, Meyer B, Ringel F: Functional preoperative and intraoperative mapping and monitoring: increasing safety and efficacy in glioma surgery. Neurosurg Focus 38(1):E3, 2015

14. Parney IF, Goerss SJ, McGee K, Huston J III, Perkins WJ, Meyer FB: Awake craniotomy, electrophysiologic mapping, and tumor resection with high-field intraoperative MRI. World Neurosurg 73:547-551, 2010

15. Sanai N, Polley MY, McDermott MW, Parsa AT, Berger MS: An extent of resection threshold for newly diagnosed glioblastomas. J Neurosurg 115:3-8, 2011

16. Senft C, Bink A, Franz K, Vatter H, Gasser T, Seifert V: Intraoperative MRI guidance and extent of resection in glioma surgery: a randomised, controlled trial. Lancet Oncol 12:997-1003, 2011

17. Smith JS, Chang EF, Lamborn KR, Chang SM, Prados MD, Cha S, et al: Role of extent of resection in the long-term outcome of low-grade hemispheric gliomas. J Clin Oncol 26:1338-1345, 2008 
18. Stummer W, Reulen HJ, Meinel T, Pichlmeier U, Schumacher W, Tonn JC, et al: Extent of resection and survival in glioblastoma multiforme: identification of and adjustment for bias. Neurosurgery 62:564-576, 2008

19. Talos IF, Zou KH, Ohno-Machado L, Bhagwat JG, Kikinis R, Black PM, et al: Supratentorial low-grade glioma resectability: statistical predictive analysis based on anatomic MR features and tumor characteristics. Radiology 239:506-513, 2006

20. Wirtz CR, Bonsanto MM, Knauth M, Tronnier VM, Albert FK, Staubert A, et al: Intraoperative magnetic resonance imaging to update interactive navigation in neurosurgery: method and preliminary experience. Comput Aided Surg 2:172-179, 1997

21. Wu JS, Gong X, Song YY, Zhuang DX, Yao CJ, Qiu TM, et al: 3.0-T intraoperative magnetic resonance imaging-guided resection in cerebral glioma surgery: interim analysis of a prospective, randomized, triple-blind, parallel-controlled trial. Neurosurgery 61 (Suppl 1):145-154, 2014

\section{Disclosures}

The authors report no conflict of interest concerning the materi- als or methods used in this study or the findings specified in this paper.

\section{Author Contributions}

Conception and design: Scherer, Jungk, Unterberg. Acquisition of data: Scherer, Younsi. Analysis and interpretation of data: Scherer, Jungk, Younsi, Kickingereder. Drafting the article:

Scherer. Critically revising the article: Jungk, Younsi, Unterberg. Reviewed submitted version of manuscript: Scherer, Jungk, Younsi, Kickingereder. Approved the final version of the manuscript on behalf of all authors: Scherer. Statistical analysis: Müller. Administrative/technical/material support: Unterberg. Study supervision: Unterberg.

\section{Correspondence}

Moritz Scherer, Department of Neurosurgery, Heidelberg University Hospital, Im Neuenheimer Feld 400, 69120 Heidelberg, Germany.email: moritz.scherer@med. uni-heidelberg.de. 